\title{
Flat Spectrum Radio Quasars through the MAGIC glasses
}

\section{J. Becerra González ${ }^{* 1,2,3}$, E. Lindfors ${ }^{4}$, E. Prandini ${ }^{5,6}$, K. Satalecka $^{7}$, J. Sitarek ${ }^{8}$, G. De Caneva $^{9}$, V. Fallah Ramazani ${ }^{4}$, F. Tavecchio ${ }^{10}$, J. McEnery ${ }^{1}$, T. Hovatta ${ }^{11,12}$, M. Tornikoski $^{11}$, A. Lähteenmäki ${ }^{11}$, S. Jorstad ${ }^{13}$ and A. Marscher ${ }^{13}$ for the the MAGIC and Fermi-LAT Collaborations}

${ }^{1}$ NASA Goddard Space Flight Center, Greenbelt, MD 20771, USA

${ }^{2}$ Department of Physics and Department of Astronomy, University of Maryland, College Park, MD 20742, USA

${ }^{3}$ Inst. de Astrofísica de Canarias, E-38200 La Laguna, Tenerife, Spain

${ }^{4}$ Finnish MAGIC Consortium, Tuorla Observatory, University of Turku and Department of Physics, University of Oulu, Finland

${ }^{5}$ ETH Zurich, $\mathrm{CH}-8093$ Zurich, Switzerland

${ }^{6}$ ISDC - Science Data Center for Astrophysics, 1290, Versoix (Geneva), Switzerland

${ }^{7}$ Universidad Complutense, E-28040 Madrid, Spain

${ }^{8}$ University of Łódź, PL-90236 Łódź, Poland

${ }^{9}$ Deutsches Elektronen-Synchrotron (DESY), D-15738 Zeuthen, Germany

10 INAF National Institute for Astrophysics, I-00136 Rome, Italy

${ }^{11}$ Aalto University Metsähovi Radio Observatory, Metsähovintie 114, FI-02540 Kylmälä, Finland

${ }^{12}$ Cahill Center for Astronomy and Astrophysics, California Institute of Technology, Pasadena, CA 91125, USA

${ }^{13}$ Institute for Astrophysical Research, Boston University, 725 Commonwealth Avenue, Boston, MA 02215, USA; Astronomical Institute

E-mail: josefa.becerra@nasa.gov

The detection of Flat Spectrum Radio Quasars (FSRQs) in the Very High Energy (VHE, E>100 $\mathrm{GeV}$ ) range is challenging, mainly because of their steep spectra in this energy band. Up to now, only five FSRQs are known to be VHE $\gamma$-ray emitters, all of them have been detected by the MAGIC telescopes, that discovered four of them in the VHE band. The observations in the VHE band are crucial to understand their emission, specially to constrain the location of the emitting region within the jet due to the absorption from their broad line region (BLR). Typically, FSRQs are detected during high flux states, enhancing the probability of detection with the current instruments sensitivities. However, the last observation campaigns performed with the MAGIC telescopes show emission during moderate states, thus challenging our understanding of the emission mechanisms in FSRQs. In this contribution, we give an overview and present the most recent results of the three FSRQs 3C279, PKS1222+21 and PKS1510-089 in a multi-wavelength context with special focus on MAGIC and Fermi-LAT simultaneous observations.

The 34th International Cosmic Ray Conference,

30 July- 6 August, 2015

The Hague, The Netherlands 


\section{Introduction}

Blazars are active galactic nuclei (AGNs) whose relativistic jets point close to our line of sight, making their emission strongly boosted. They constitute the most numerous source class detected at high energies, dominating the extragalactic $\gamma$-ray sky. Only 65 extragalactic sources have been detected to date in the VHE band by the Cherenkov telescopes, 59 of them being blazars. Blazars emit strongly throughout the whole electromagnetic spectrum, and their multi-wavelength (MWL) spectral energy distributions (SEDs) are characterized by two broad peaks. Depending on the frequency of the peaks and the optical spectral characteristics, blazars are classified as BL Lac objects and flat spectrum radio quasars (FSRQs). The MWL SED of FSRQs typically peaks at lower energies [1], making the detection of such targets challenging in VHE. Up to now, only 5 FSRQs have been detected in the VHE band: 3C 279 [2, 3], PKS 1510-089 [4, 5], PKS 1222+216 [6], S3 0218+357 [7] and PKS 1441+25 [9, 10].

The emission of blazars at low frequencies is attributed to synchrotron emission from relativistic electrons in the jet. The high energy peak origin is still under debate, it can be produced through inverse Compton scattering within a low frequency photon field (leptonic models) or by hadronic interactions [14]. The low frequency photon field can have different origins: the synchrotron emission within the jet (Synchrotron self-Compton -SSC- models, [11]) or an external region to the jet, the optical-UV photons from the BLR or the IR photons from the dusty torus (External Compton models, [12], [13]). In FSRQs, typically the high energy peak is much more luminous that the low energy one (Compton dominance). In such case, it is hard to explain the high energy emission only as SSC, and some additional external seed photons are needed to explain its SED. Strong emission lines are typically present in the optical spectra from FSRQs, we can infer therefore that there is a strong BLR in this type of blazars, with high density of UV-optical photons. Such UV-optical photons can serve as seed for the inverse Compton process to produce high energy photons. However, it can also contribute to the absorption of the $\gamma$-rays. If the emitting region of the jet is located within the BLR, a strong absorption is expected above tens of $\mathrm{GeV}$, in the high energy extreme of the Fermi-LAT energy range. Instead, if the emitting region within the jet is considered to be located farther away from the central engine, were the IR photons form the torus are dominant as external seed for the inverse Compton process, the absorption spectral feature is expected to be detected above $\sim 1 \mathrm{TeV}$. Therefore, VHE observations are crucial to constrain the location of the emitting region in FSRQs together with the Fermi-LAT monitoring.

The FSRQs detected in VHE band show different observational characteristics. A brief description of each FSRQ detect in the VHE band up to date can be found below:

- 3C $279(\mathrm{z}=0.54)$ has been detected twice in VHE $\gamma$-rays before 2014: MAGIC discovered the source on February 23, 2006, and then detected it again on January 16, 2007. The flux and spectra of the two observations are comparable within the errors, the 2006 measured integral flux being $\mathrm{F}(>150 \mathrm{GeV})=\left(5.15 \pm 0.82_{\text {stat }} \pm 1.5_{\text {syst }}\right) \times 10^{-10} \mathrm{phcm}^{-2} \mathrm{~s}^{-1}$ and spectral index $\alpha=4.1 \pm 0.7_{\text {stat }} \pm 0.2_{\text {syst }}$. In both cases, the significant detection is preceded by a hint of signal on 2-3 $\sigma$ level from the previous night.

\footnotetext{
* Speaker.
} 
- PKS 1222+216 ( $\mathrm{z}=0.43$ ) was detected in VHE $\gamma$-rays on June 17, 2010 by the MAGIC telescopes. The source had been monitored by the MAGIC telescope since the beginning of May 2010, the data from May 8, 2010 showed a hint of signal at $4.4 \sigma$ level [15]. The MAGIC observations on June 17, 2010 had a duration of only 30 minutes, but even within this short observation the flux was significantly variable with the flux doubling time of order of $\sim 9$ minutes [6]. The observed spectral slope is soft with $\Gamma=3.75 \pm 0.29$. In 2014, VERITAS reported the detection of a significant signal from this source from several nights rather than from a single night strong flare [16]. This detection is coincident with the MAGIC observations discussed in this contribution.

- PKS 1510-089 $(\mathrm{z}=0.36)$ has also been detected twice in VHE $\gamma$-rays in two extended periods from March and April 2009 by H.E.S.S. [4] and February-April 2012 by MAGIC [5]. The flux and spectra of the two observations agree within the errors. The integral flux of the 2012 measurement is $\mathrm{F}(>200 \mathrm{GeV})=(3.6 \pm 0.9) \times 10^{-11} \mathrm{ph} \mathrm{cm}^{-2} \mathrm{~s}^{-1}$. The best-fit power law to the VHE data has a photon index of $\Gamma=3.8 \pm 0.4_{\text {stat }} \pm 0.3_{\text {sys. }}$. There is no published upper limits of the source below these detections and therefore it cannot be concluded if the detected flux presents high or quiescent state of the source at VHE $\gamma$-rays. Neither of the data sets show statistically significant variability on daily, weekly or monthly scales. It should be noted, however, that in the Fermi Hard source catalog this source is listed as variable above $>10$ $\mathrm{GeV}$ [17].

- S3 0218+357 is a gravitationally lensed FSRQ discovered by MAGIC in July 2014 [7] at the redshift of 0.94. The VHE detection was possible thanks to the gravitational lens effect from which we could predict when the delayed emission from the flare would reach the Earth based on a previous Fermi-LAT flare detection [8].

- PKS 1441+25 is the latest FSRQ detected in the VHE band, first discovered by MAGIC [9] and later confirmed also by VERITAS [10]. It is also a very distant blazar located at the same distance than S3 0218+357, z=0.94. The observations were triggered by the high state and specially the hard index observed by the Fermi-LAT.

S3 $0218+357$ and PKS $1441+25$ are the most distant VHE blazars known to date. Besides the study of the intrinsic characteristics of FSRQs, which are poorly known due to the lack of statistics, they are also important to test for the first time the EBL models at high redshifts in this energy range.

As discussed above, due to the steep spectrum of FSRQs in the VHE band, they are typically detected while undergoing strong flares. However, in this contribution we will present the results from the observational campaign in 2013-2014 of the three VHE FSRQs 3C 279, PKS 1510-089 and PKS $1222+216$. Their detection in the VHE band is not strongly correlated with the high state of the sources.

\section{MAGIC data analysis and results}

The VHE $\gamma$-ray observations were performed by the MAGIC telescopes. The MAGIC system consists of two $17 \mathrm{~m}$-diameter Imaging Atmospheric Cherenkov Telescopes (IACT) located on the 


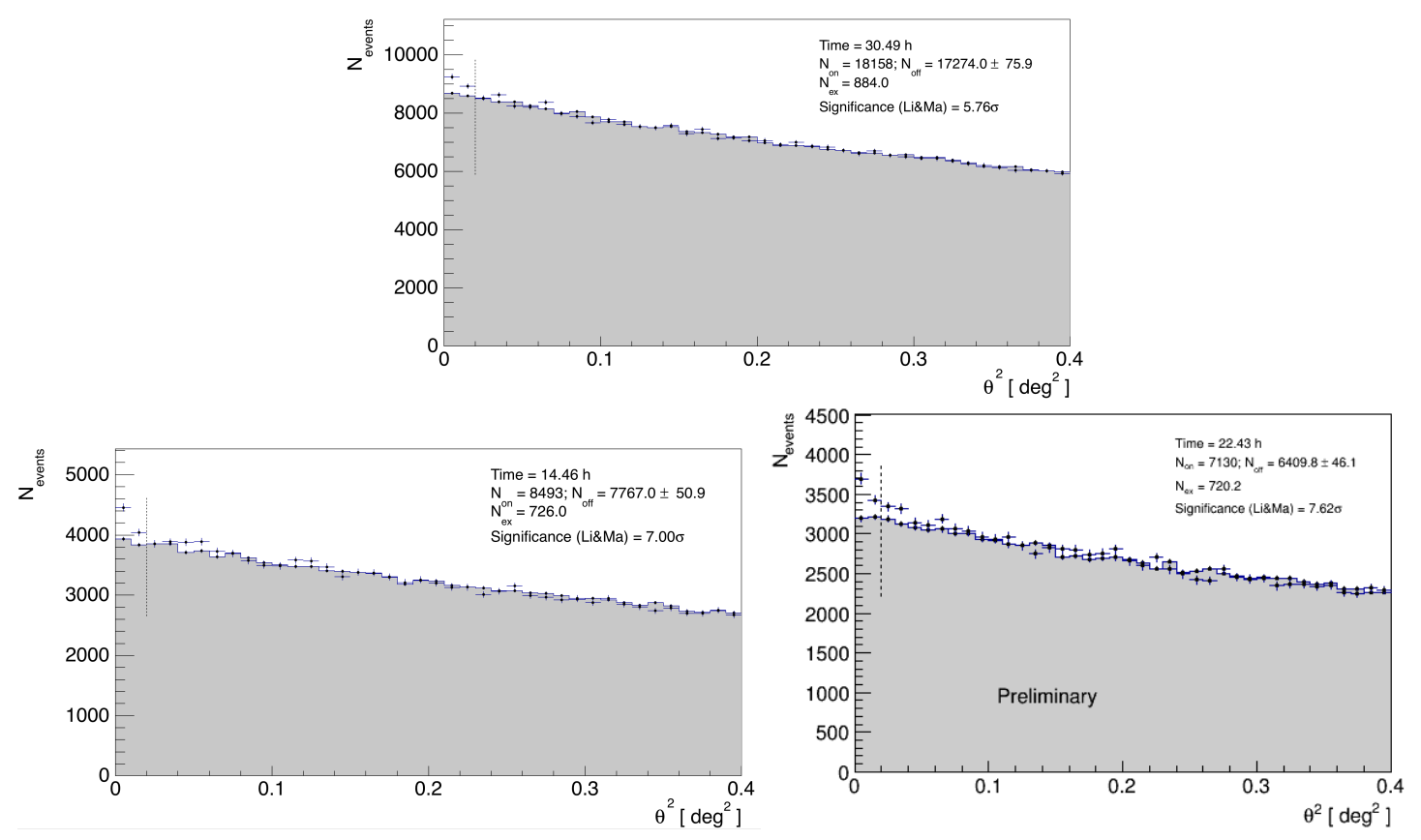

Figure 1: Detection plot for the FSRQs 3C 279, PKS 1222+216 and PKS1510-089 during 2013-2014 campaign with the MAGIC telescopes. $\theta^{2}$ is calculated as the square distance between the reconstructed and nominal position of the source. Black points represent the $\theta^{2}$ distribution from the source, while the distribution for background events are denoted by the gray shaded area.

Roque de los Muchachos, Canary Island of La Palma $\left(28^{\circ} 46^{\prime} \mathrm{N}, 17^{\circ} 53^{\prime} \mathrm{W}\right)$, at a height of $2200 \mathrm{~m}$ above sea level. The system reaches a sensitivity of $(0.66 \pm 0.03) \%$ of the Crab Nebula flux for $\mathrm{E}>220 \mathrm{GeV}$ in $50 \mathrm{~h}$ of observations [19].

The VHE observations of the three FSRQs we discuss in this contribution where observed with the MAGIC telescopes in wobble mode [20], with the source located $0.4^{\circ}$ from the centre of the field of view. The analysis of the data has been performed using the standard MAGIC analysis chain $[21,22]$.

The FSRQ 3C279 was observed for 30.5 hours from 2013-12-30 to 2014-05-31 (MJD 5665656808 ) with zenith angles from 34 to 50 degrees. As shown in Fig. 1, the source was detected with a confident level of $5.8 \sigma$.

The observations on PKS 1222+216 were carried out from 2013-12-30 to 2014-03-25 (MJD 56656-56741) at zenith angles from 9 to 46 degrees for a total of 14.46 hours. We detected the source at $7.0 \sigma$ level.

PKS 1510-089 was observed with the MAGIC telescopes from 2013-03-15 to 2013-06-05 (MJD 56366-56448) and 2014-06-17/18 (MJD 56825/26) for 22.4 hours covering a zenith angle range from 27 to 45 . The plot shown in Fig. 1 reveals a detection of the source at $7.6 \sigma$ confidence level.

The nightly light curve at $\mathrm{E}>150 \mathrm{GeV}$ does not show hints of variability for any of the three sources presented in this contribution. The flux is compatible with a constant emission during the observed period. 


\section{Multi-wavelength framework and results}

Since blazars emit throughout the whole electromagnetic spectrum and are highly variable sources, they can only be properly studied in a MWL framework. Only part of the large compilation of MWL data will be presented in the proceedings, but they will be discussed in the oral contribution and the forthcoming paper.

Thanks to the strong cooperation between the Fermi-LAT and the MAGIC telescopes together with the optical monitoring performed with the KVA telescope by the Tuorla Observatory AGN group (http://users.utu.fi/kani/1m/), we have detected the "classical" VHE FSRQs during moderate gamma-ray activity and two new VHE FSRQs have been discovered with the MAGIC telescopes. The long-term monitoring of the gamma-ray sky performed by Fermi-LAT above $100 \mathrm{MeV}$ allows to study in detail the time evolution of the emission from individual sources. This is specially important in the case of FSRQs. As discussed in the previous sections, due to the intrinsic characteristics of FSRQs, the detection of the VHE gamma-rays is very challenging. However, Fermi-LAT observations can trigger ground-based Cherenkov telescopes during active periods of the source. This is particularly relevant when considering the small field of view and short duty cycle of the Cherenkov telescopes.

In Fig. 2, the gamma-ray light curve of 3C279 above $100 \mathrm{MeV}$ observed with the Fermi-LAT and the optical R-band light curve observed with the KVA telescope are shown. The vertical lines denote the MAGIC observations. The flare around MJD 56750 detected with the LAT is one of the strongest flare of the source detected with this instrument. Unfortunately, the peak of the emission was not well sampled by the MAGIC telescopes due to moon/weather constraints. Nevertheless, the VHE emission is not centered only around the strong flare. Indeed, it is compatible with a constant flux during the observation period. There is not clear correlation between optical and gamma-rays evolution.

In case of PKS 1222+216, as show in Fig. 3, due to observation constraints MAGIC could not observe the strong flare during the period. As opposite to the case of $3 \mathrm{c} 279$, the gamma-ray and optical seem to be correlated at least during the strong flare. However, the optical flare is delayed with respect to the gamma-ray.

The FSRQ PKS 1510-089 was only detected by MAGIC during the 2013 campaign, therefore, the light curve shown in Fig. 4 is focuses in that period. As in the case of 3c279, no strong correlation can be claimed between the optical and gamma-ray light curves. MAGIC observations were only performed during moderate activity of the source in gamma-rays.

Despite the different characteristics of the sources and evolutions with/without gamma-ray and optical correlation, all three sources were detected during moderate gamma-ray activity. No hint of variability in the MAGIC light curves was found. The only previous detection compatible with a constant emission was PKS 1510-089, for the rest of the FSRQs, strong variability has been previously one of the fingerprints of FSRQs detection in the VHE band. However, remarkably during the 2013-2014 VHE observation campaign, the detection of the three sources are compatible with a constant flux, pointing to the fact that the VHE emission in FSRQs not necessarily need to be associated with extreme flares. 

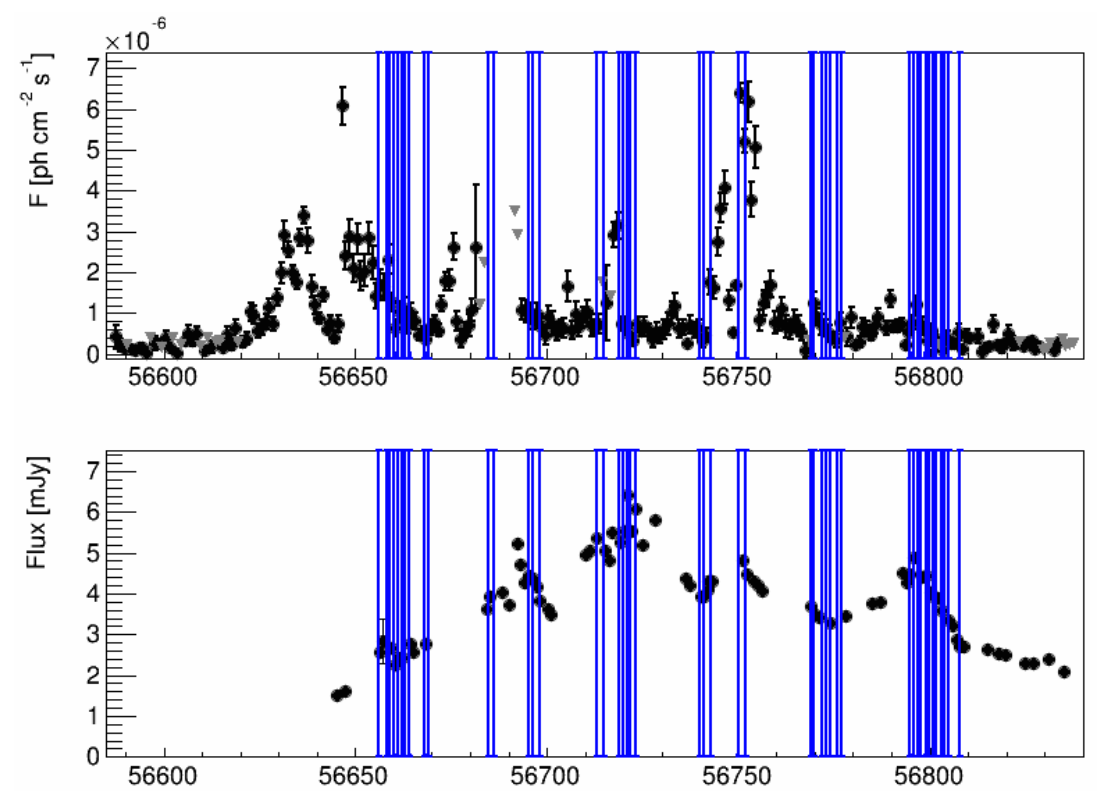

Figure 2: Top: Light curves of the FSRQ 3c279 for energies between $100 \mathrm{MeV}$ and $300 \mathrm{GeV}$ observed with the Fermi-LAT and calculated in 1 day bins. Bottom: R-band light curve observed with the KVA telescope. The vertical lines denote the MAGIC observations.
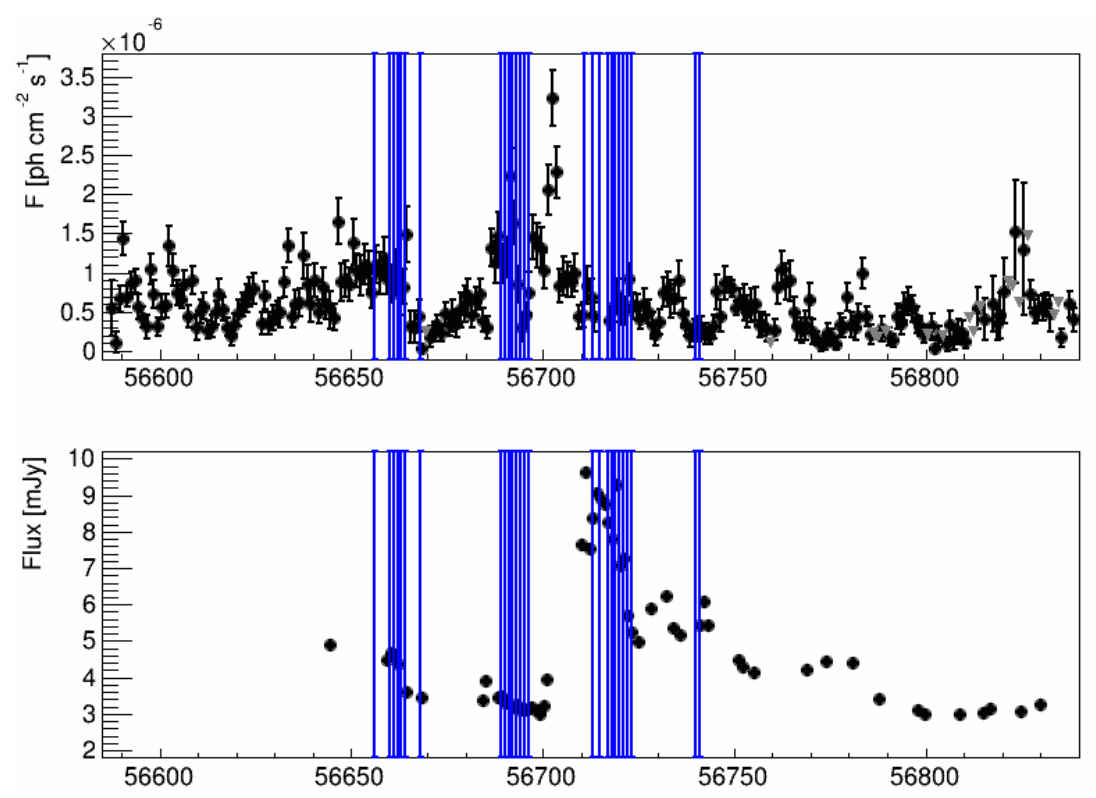

Figure 3: Top: Light curves of the FSRQ PKS 1222+216 for energies between $100 \mathrm{MeV}$ and $300 \mathrm{GeV}$ observed with the Fermi-LAT and calculated in 1 day bins. Bottom: R-band light curve observed with the KVA telescope. The vertical lines denote the MAGIC observations. 

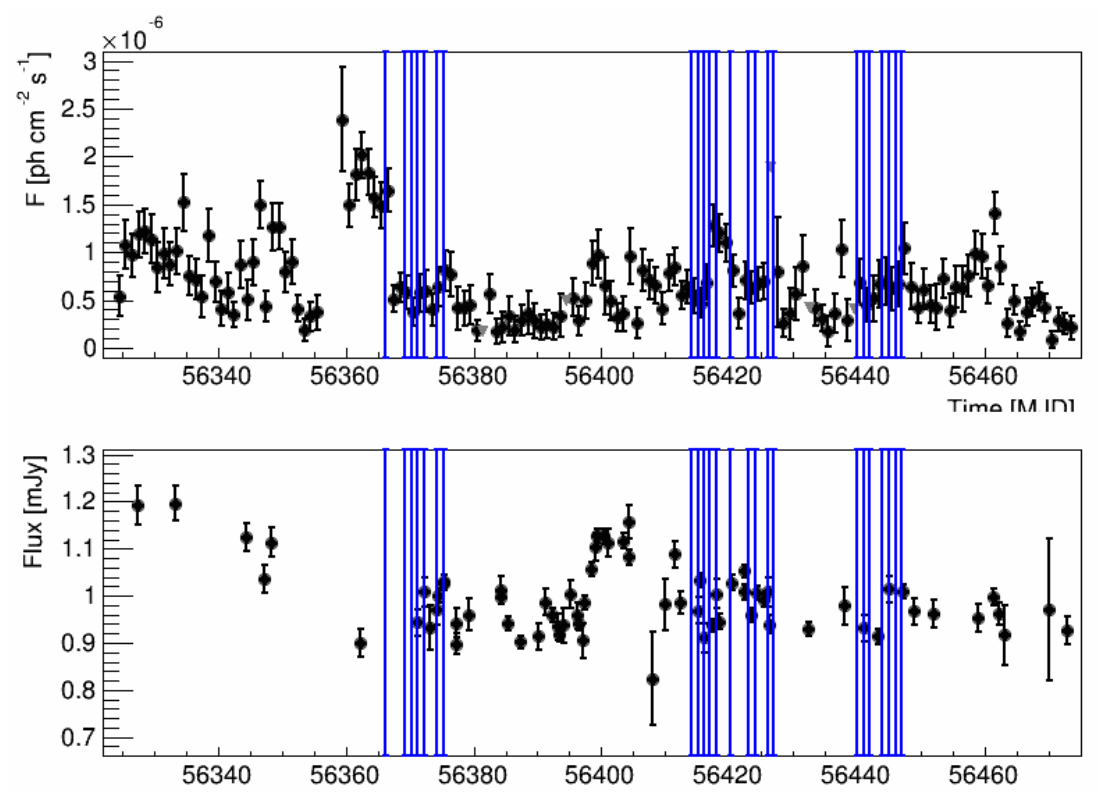

Figure 4: Top: Light curves of the FSRQ PKS 1510-089 (only 2013 data) for energies between $100 \mathrm{MeV}$ and $300 \mathrm{GeV}$ observed with the Fermi-LAT and calculated in 1 day bins. Bottom: R-band light curve observed with the KVA telescope. The vertical lines denote the MAGIC observations.

\section{Acknowledgments}

We would like to thank the Instituto de Astrofísica de Canarias for the excellent working conditions at the Observatorio del Roque de los Muchachos in La Palma. The financial support of the German BMBF and MPG, the Italian INFN and INAF, the Swiss National Fund SNF, the ERDF under the Spanish MINECO (FPA2012-39502), and the Japanese JSPS and MEXT is gratefully acknowledged. This work was also supported by the Centro de Excelencia Severo Ochoa SEV-2012-0234, CPAN CSD2007-00042, and MultiDark CSD2009-00064 projects of the Spanish Consolider-Ingenio 2010 programme, by grant 268740 of the Academy of Finland, by the Croatian Science Foundation (HrZZ) Project 09/176 and the University of Rijeka Project 13.12.1.3.02, by the DFG Collaborative Research Centers SFB823/C4 and SFB876/C3, and by the Polish MNiSzW grant 745/N-HESS-MAGIC/2010/0.

The Fermi-LAT Collaboration acknowledges support for LAT development, operation and data analysis from NASA and DOE (United States), CEA/Irfu and IN2P3/CNRS (France), ASI and INFN (Italy), MEXT, KEK, and JAXA (Japan), and the K.A. Wallenberg Foundation, the Swedish Research Council and the National Space Board (Sweden). Science analysis support in the operations phase from INAF (Italy) and CNES (France) is also gratefully acknowledged. 


\section{References}

[1] G. Fossati et al., $A$ unifying view of the spectral energy distributions of blazars, MNRAS 98, 299, 433

[2] J. Albert et al., Very-High-Energy gamma rays from a Distant Quasar: How Transparent Is the Universe?, Science 08, 320, 1752

[3] J. Aleksic̀ et al., MAGIC observations and multiwavelength properties of the quasar 3C279 in 2007 and 2009, A\&A 11, 530, id.A4

[4] A. Abramowski et al., H.E.S.S. discovery of VHE ?-rays from the quasar PKS 1510-089, A\&A 13, 554, id.A107

[5] J. Aleksic̀ et al., MAGIC gamma-ray and multi-frequency observations of flat spectrum radio quasar PKS 1510-089 in early 2012, A\&A 14, 569, id.A46

[6] J. Aleksic̀ et al., MAGIC Discovery of Very High Energy Emission from the FSRQ PKS 1222+21, ApJL 11, 730, L8

[7] R. Mirzoyan, Discovery of Very High Energy Gamma-Ray Emission From Gravitationally Lensed Blazar S3 0218+357 With the MAGIC Telescopes, ATel \#6349

[8] C. C. Cheung et al., Fermi Large Area Telescope Detection of Gravitational Lens Delayed ?-Ray Flares from Blazar B0218+357, ApJ, 782, L14

[9] R. Mirzoyan, Discovery of Very High Energy Gamma-Ray Emission from the distant FSRQ PKS $1441+25$ with the MAGIC telescopes, $A$ Tel \#7416

[10] R. Mukherjee, Very-high-energy gamma-ray emission from PKS 1441+25 detected with VERITAS, ATel \#7433

[11] L. Maraschi et al., A jet model for the gamma-ray-emitting blazar 3C279, ApJ 92, 397, L5

[12] Dermer C. D. et al., High-energy gamma radiation from extragalactic radio sources, A\&A,92, 256, L27

[13] Ghisellini G., \& Madau P., On the origin of the gamma-ray emission in blazars, MNRAS, 96, 280, 67

[14] M. Böttcher et al., Leptonic and Hadronic Modeling of Fermi-detected Blazars, ApJ, 13, 768, id. 54

[15] M. Ackermann et al., Multifrequency Studies of the Peculiar Quasar 4C +21.35 During the 2010 Flaring Activity, ApJ 14,786, id. 157

[16] J. Holder, Detection of Persistent VHE emission from PKS 1222+216 (4C +21.35) with VERITAS, ATel \#5981

[17] M. Ackermann et al., The First Fermi-LAT Catalog of Sources Above $10 \mathrm{GeV}$, ApJS 13, 209, id. 34

[18] S. Buson, Fermi LAT Detection of a Hard Spectrum Gamma-ray Flare from Gravitationally Lensed Blazar S3 0218+357, ATel \#6316

[19] J. Aleksic̀ et al., The major upgrade of the MAGIC telescopes, Part II: A performance study using observations of the Crab Nebula, arXiv:1409.5594

[20] V. P. Fomin et al., New methods of atmospheric Cherenkov imaging for gamma-ray astronomy. I. The false source method, $A$ pJ 94, 2, 137

[21] A. Moralejo et al., MARS, the MAGIC Analysis and Reconstruction Software, [arXiv:0907.0943]

[22] S. Lombardi et al., Advanced stereoscopic gamma-ray shower analysis with the MAGIC telescopes [arXiv:1109.6195] 